\title{
Teoría específica sobre las conductas familiares de las personas con diabetes tipo 2
}

López-González, Juan Alberto*; Bañuelos-Barrera, Yolanda²; Ruíz-Paloalto, Laura3; Álvarez-Aguirre, Alicia³

\section{RESUMEN}

Introducción: Los profesionales de enfermería desarrollan Teorías sobre situaciones específicas para tener una mejor explicación de la práctica y poder brindar un cuidado de calidad a la persona, la familia y la comunidad. Objetivo: Describir la Teoría específica sobre las Conductas familiares en la Autogestión y el Control glucémico de las personas con Diabetes tipo 2. Metodología: El método utilizado para el desarrollo de está teoría fue a través del Proceso integrador. Resultados: se logró identificar cuatro conceptos principales: Conductas familiares, Autogestión de la persona con Diabetes tipo 2, Control glucémico y Agentes condicionantes individuales. Conclusiones: la Teoría específica desarrollada, nos permite tener un marco teórico para entender la interacción del familiar y la persona con Diabetes tipo 2 para el logró de la Autogestión y el Control glucémico de estás.

Palabras claves: Teoría de Enfermería; Apoyo social; Autogestión; Diabetes Mellitus (DeCS;BIREME).

${ }^{1}$ Estudiante del Doctorado en Ciencias de Enfermería de la Universidad de Guanajuato, Becario de la Universidad de Sonora y del Centro Médico Dr. Ignacio Chávez (ISSSTESON); México. E-mail: juan.lopez@unison.mx.

²Doctora en Ciencias de Enfermería; Profesora de Tiemplo Completo de la Facultad de Enfermería y Obstetricia de la Universidad de Juárez del Estado de Durango.

${ }^{3}$ Doctora en Ciencias de Enfermería; Profesora del Departamento de Enfermería Clínica de la Universidad de Guanajuato, Campus Celaya-Salvatierra.

*Autor para correspondencia

\section{Cómo citar este artículo}

López-González JA, Bañuelos-Barrera Y, Ruiz-Paloalto L, Álvarez-Aguirre A Teoría específica sobre las conductas familiares de las personas con diabetes tipo 2. Sanus. 2018;3(7): 40-55. [Acceso ]; Disponible en: mes día año URL 


\section{INTRODUCCIÓN}

La Diabetes Tipo 2 (DT2) es uno de los principales problemas de salud del siglo XXI, está impacta de forma importante en la calidad de vida de las personas que la padecen; en las últimas décadas la incidencia y prevalencia de la enfermedad se ha incrementado de manera exorbitante a nivel global. La Organización Mundial de la Salud (OMS) y la Federación Internacional de Diabetes (FID) la consideran ya como una pandemia, la cual afecta a la mayoría de los países del mundo(1,2).

Según reportes de la FID en el año 2015, más del 8.8\% de la población adulta de 20-79 años (415 millones) padecían la enfermedad (3), mientras que la OMS en el 2016 reportó cerca de 442 millones de personas en este grupo. El incremento de esta enfermedad continua año con año y de no controlarse se estima que para el 2040, a nivel mundial habrá 642 millones de personas con este padecimiento(2).

En México la situación no es diferente, ya que es el sexto país con mayor número de personas con DT2 (11.5 millones) a nivel mundial, y de no tomar medidas que ayuden a prevenir la enfermedad, se espera que en el 2040 pase a ocupar la quinta posición ${ }^{(3)}$. La Encuesta Nacional de Salud y Nutrición (ENSANUT)(4), en el 2016 reportó el $9.4 \%$ de la población mexicana padecen la DT2, cuyo diagnóstico se realiza cada vez a más temprana edad. De igual forma, la reportan como una de las principales causas de demanda de los servicios de salud y de mayor discapacidad prematura en la población, lo que representa una carga económica para la persona que la padece y para la sociedad en general(5).

Existe evidencia de que el padecimiento es resultado de los cambios culturales y sociales que se están viviendo en la actualidad, como el envejecimiento de la población, incremento de la urbanización, nuevas dietas alimenticias, disminución de la actividad física y empleos más sedentarios ${ }^{(6)}$. Se considera que dicho padecimiento requiere de una atención, educación, apoyo continuo para prevenir el desarrollo de la enfermedad y el retraso las complicaciones y sobre todo la mortalidad prematura de las personas afectadas ${ }^{(7)}$.

Vivir con DT2 y sus complicaciones (agudas y crónicas), representa muchos desafíos para la persona, que van desde un apego a regímenes terapéuticos y la implementación de cambios en los comportamientos, encaminados a mejorar el control glucémico ${ }^{(8)}$. Además experimentan continuamente una situación difícil y estresante en sus vidas como consecuencia de llevar a cabo estos cambios para controlar y retrasar las complicaciones agudas y crónicas de la enfermedad ${ }^{(9,10)}$. Dicho estrés puede tener un impacto negativo, que podría repercutir en el manejo apropiado de la enfermedad(11). Por ello, el afrontamiento de la enfermedad es una parte primordial para que las personas lleven a cabo de manera correcta, de acuerdo con las diversas situaciones presentadas por la enfermedad a lo largo de su vida. Se entiende como el afrontamiento de la DT2, aquellos procesos de cambio cognitivo y conductuales que realiza la persona para hacer frente a situaciones estresantes, cuyo propósito es adaptarse al entorno social(11,12).

La autogestión es la clave para lograr un control del padecimiento, lo que contribuiría de manera significativa al retraso de las complicaciones y mejoramiento de la calidad de vida de las personas a corto y largo plazo ${ }^{(7,13)}$. Ésta es entendida como aquellas acciones o actividades realizadas por la persona, encaminadas al monitoreo de signos y síntomas (manejo de hipoglucemias e hiperglucemias, y el cuidado de los pies) el mantenimiento y la mejora de los comportamientos de la salud (mejorar sus patrones de alimentación, actividad física, medicación, abandono del consumo de alcohol y tabaco) y la disminución del impacto negativo de la enfermedad (manejo del estrés, de complicaciones y efectos secundarios) ${ }^{(7,14)}$.

Dicha autogestión se ve influenciada por diversos factores, como el físico, psicológico, cultural y social $(15,16)$. En este caso, la autoeficacia juega un papel muy importante en el manejo de la enfermedad, debido a que requiere que las personas confíen en sus capacidades para el manejo y control de ésta. Entendiéndose a la autoeficacia como las creencias que tiene la persona con DT2 para llevar a cabo un comportamiento y superar las barreras con el fin de lograr una meta específica (control glucémico y retraso de complicaciones) ${ }^{(17,18)}$.

Cumplir y mantener estos complejos comportamientos es especialmente difícil, cuando no se cuenta con un apoyo, debido a que muchas de las actividades requieren de la ayuda y colaboración de la familia, amigos y de los proveedores de salud ${ }^{(18,19)}$. Se ha visto que el Apoyo Social (AS) refuerza e influye de manera positiva los comportamientos en la autogestión, el afrontamiento y la autoeficacia de las personas con DT $2^{(13,20)}$. Se entiende por AS como aquellas relaciones interpersonales de al menos dos o más personas, entre las cuales se lleva el intercambio reciproco de recursos tangibles e intangibles, cuyo propósito es amortiguar los factores estresantes de la enfermedad y el ambiente ${ }^{(21-24)}$.

El contexto familiar es un determinante importante para que las personas con DT2 lleven a cabo las acciones de autogestión de la enfermedad de manera eficaz, teniendo en cuenta que la función principal de la familia es satisfaccer las necesidades individuales de sus miembros. El funcionamiento familiar, es un aspecto importante en el entorno familiar que puede ayudar o afectar en la calidad de vida de estas personas con problemas crónicos. Se ha logrado identificar que en los casos de un buen funcionamiento en las interaciones familiares, existe cambios graduales en los estilos de vida, aumento del bienestar y ayuda para enfrentar las situaciones estresantes de la enfermedad $(25,26)$. La asistencia proporcionada incluye ayudar al individuo 
a preparar alimentos saludables, estimularlo para que tome sus medicamentos en tiempo y forma, realizar actividades físicas de manera individual o en grupo, y por último, facilitando el acceso a los profesionales de salud médico, enfermera, psicólogo, nutriólogo, etc.) $(13,18,27)$. Pero en los casos de un mal funcionamiento de la familiar, se asocian a peor control glucémico, presencia de estrés y depresión en las personas con DT2 ${ }^{(25)}$.

A lo largo de la historia, diferentes teóricas de enfermería, han construido conocimiento de enfermería, desarrollando modelos y teorías que permitan describir, explicar y predecir el fenómeno de interés de la disciplina, esto con el propósito de distinguirse de otras disciplinas encargadas de brindar el cuidado a la persona ${ }^{(28)}$. En las últimas décadas se ha dado un auge en el desarrollo de teorías de enfermería más aplicativas en el entorno practico de la enfermería, estas reciben el nombre de Teorías Específicas sobre la situación (Microteorías), las cuales tratan de describir los fenómenos de una manera más específica o práctica, son menos abstractas que las grandes teorías o modelos de enfermería y pueden derivarse de una teoría de rango medio, pero diversos autores refieren que también surgen de Modelos y de Grandes Teorías ${ }^{(29,30)}$. El objetivo del presente artículo es describir la Teoría específica sobre las Conductas familiares en la Autogestión y el Control glucémico de las personas con Diabetes tipo 2.

\section{METODOLOGÍA}

La Teoría Específica sobre las Conductas familiares para la Autogestión y el Control glucémico de las personas con Diabetes tipo 2, fue desarrollada a partir del Enfoque Integrador (EI) propuesto por Im et al. (31,32), el cual esta compuesto por cuatro etapas: 1) la comprobación de suposiciones para el desarrollo de la teoría (2), la exploración del fenómeno a través de múltiples fuentes (3), la teorización y por último (4) el informe, compartir y validar. A continuación se describe el proceso realizado para el desarrollo de la presente microteoría, el cual se puede ver en la Figura 1.

Figura 1. Proceso para el desarrollo de la Teoría Especifica sobre las Conductas familiares para la Autogestión y el Control glucémico de las personas con Diabetes tipo 2

\section{Paso 1. Suposiciones}

Las Conductas familiares contribuyen a que las personas con Diabetes lleven a cabo acciones para el control de la enfermedad.

Para que la persona con Diabetes lleve actividades para cuidar de su enfermedad, debe de tomar decisiones continuamente y tener confianza en si mismo de lograr las metas impuestas por la enfermedad.

El control de la enfermedad dependerá del apoyo familiar y de las acciones realizadas por la persona con la enfermedad.

Hay factores tanto internos como externos que contribuyen al control de la enfermedad.

Paso 2. Múltiples fuentes

Teoría de Apoyo social

Revisión de evidencia empírica

PubMed

EBSCO Host

Criterios de inclusión:

Idioma inglés

Publicado en los últimos 5 años en relación

Los descriptores utilizados fueron "Diabetes Mellitus", "Apoyo social" y "Autogestión"

Artículos de revistas

Paso 3. Teorización

Se obtuvieron cuatro conceptos principales posterior al análisis de la evidencia empírica:

Conductas Familiares

Autogestión de las personas con Diabetes tipo 2

Control glucémico

Agentes condicionantes individuales

Paso 4. Reporte, compartir y validar

Fuente: Elaboración propia 


\section{Descripción de pasos del El}

\section{Primer paso: Suposiciones}

Para explicar el primer paso del El se identificaron y verificaron varias suposiciones. La primer suposición, las conductas llevadas a cabo por los miembros de la familia contribuyen a que las personas con DT2 lleven acciones o no, para poder controlar la enfermedad. La segunda suposición, para llevar a cabo la Autogestión se requiere que la persona tenga la capacidad de afrontar y tomar decisiones de manera continua, de acuerdo con las demandas de la enfermedad; también debe tener confianza para lograr las metas impuestas por los profesionales de la salud. La tercera suposición, el control de la enfermedad dependerá de las acciones llevadas a cabo por la persona enferma y del apoyo recibido por sus familiares, en la interacción en su contexto familiar. La cuarta suposición establece que existen agentes que condicionan las Conductas familiares, la Autogestión y el Control glucémico de las personas con DT2.

\section{Segundo paso: Múltiples fuentes}

Para el desarrollo de la teoría específica se utilizaron: a) la Teoría de apoyo social, b) una revisión de evidencia empírica en múltiples base de datos.

\section{Descripción de la Teoría de apoyo social}

La Teoría Apoyo Social (TAS) aborda la estructura y la función de las relaciones interpersonales de al menos dos personas, cuya finalidad es el intercambio de apoyo tangible e intangible, de manera recíproca y en un contexto de estrés $(23,24,33)$. Los primeros teóricos en mencionar y trabajar la TAS son Cassel ${ }^{(34)}$ y Cobb $^{(34)}$, declaran que es un factor protector y facilitador del afrontamiento y la adaptación ante eventos estresantes del entorno, cuyo objetivo final es el bienestar de las personas. Por su parte Cohen, et al.,(21) refiere que el estrés, es producido cuando una persona percibe una situación amenazante (estímulos internos o externo) y no cuenta con una respuesta de afrontamiento adecuado a dicha situación.

La TAS ha sido analizada desde la perspectiva estructural y funcional. La visión estructural analiza el apoyo brindado a través de las redes sociales(RS), entendiéndose estas como las relaciones especificas entre el individuo focal (receptor de apoyo social) y la otra persona de la red (proveedor del apoyo social ) ${ }^{(35)}$. El apoyo proporcionado puede ser de manera formal (profesionales de la salud) e informal (familia, amigos, grupos de ayuda mutua). Esta estructura la podemos describir como una característica diádica; la RS tiene diversas funciones que van desde una influencia social, control social, compañerismo social, apoyo social y socavamiento ${ }^{(23,35)}$.
La visión funcional aborda el intercambio de recursos tangibles (no psicológicos) e intangibles (psicológicos) en la relación interpersonal, los cuales pueden ser de cuatro tipos: Emocional, que consiste en aquellos actos reconfortantes (cuidado, empatía, cariño, amor, preocupación y confianza), pretende aliviar la incertidumbre, la ansiedad, la desesperanza y la depresión, es la categoría más importante a través de la cual se transmite la percepción del apoyo. El informativo es el asesoramiento, sugerencia e información, se proporciona para la resolución de problemas, en una situación de estrés. La evaluación (retroalimentación) consiste en la transmisión de información y retroalimentación para llevar a cabo la autoevaluación, a través de la comparación social. Finalmente, el instrumental es la provisión de ayuda tangible o directa a la persona en situación de necesidad, esta puede ser a través de bienes (ayuda económica o material) o de servicios (cuidado a la persona, ayudar a la realización de trabajo asignado para otros) $^{(22,23,36) \text {. }}$

\section{Revisión de la literatura}

Para la exploración del conocimiento actual sobre el AS en la Autogestión de las personas con Diabetes tipo 2, se llevo una revisión de la evidencia empírica esto en varias bases de datos (Pub Med y EBSCO Host). Los artículos incluidos para el desarrollo de esta teoría específica, se guio con los siguientes criterios: a) estuvieran en idioma ingles, b) publicado en los últimos 5 años en relación, c) los descriptores utilizados fueron "Diabetes Mellitus", "Apoyo social" y "Autogestión", y d) artículos de revistas. Se encontraron 452 artículos de los cuales se identificaron 7 que reunían los criterios y permitió desarrollar está teoría.

\section{Tercer paso: Teorización}

Desarrollo de la Teoría Específica sobre las Conductas familiares para la Autogestión y el Control glucémico de las personas con Diabetes tipo

Las personas son entidades que interactúan en un entorno y responden a múltiples factores estresantes. Está interacción es fundamental de toda persona, para logra la búsqueda y provisión de apoyo para enfrentar los cambios del ambiente ${ }^{(37)}$. Las relaciones sociales son el fundamento de la existencia humana, el nivel interpersonal, es la clave para entender e intervenir en las relaciones. La relación en el nivel interpersonal involucra la relación con los miembros de la familia, amigos, compañeros, vecinos y proveedores de salud. La relación familiar, es la principal y más importante de estas relaciones, ya que en ella se provee la ayuda (informativa, emocional, instrumental y evaluación) a los individuos para enfrentar los factores estresantes del entorno, con 
ello se logrará un bienestar de la parte receptora. Estas interacciones son de aspecto positivo, pero también se ha logrado identificar interacciones negativas, en donde el apoyo brindado, no logra el bienestar adecuado de la persona receptora ${ }^{(38)}$.

Las personas con DT2 enfrentan continuamente una situación estresante, ya que el padecimiento les demanda un cambio continuo en sus patrones de comportamiento, esto para lograr un control glucémico y un retraso en sus complicaciones crónicas ${ }^{(10)}$. Las acciones realizadas por la persona (una alimentación equilibrada, incremento de la actividad física, adherencia a terapia medicamentosa, un monitoreo de la glucosa, revisión periódica de pies, abandono de alcohol y cigarro, etc.), son la clave para controlar la enfermedad y mejorar su calidad de vida, a corto y largo plazo ${ }^{(8,17)}$. El cumplimiento y mantenimiento de estas complejas actividades, es especialmente difícil, si no se cuenta con apoyo, ya que muchas de estas actividades requieren de la colaboración por parte de la familia para lograrlas (preparación de alimentos equilibrados, acompañamiento en la actividad física, recordatorios para la citas con profesionales de la salud, etc. $)^{(13,39)}$.

La Teória específica sobre las Conductas familiares para la Autogestión y el Control glucémico de las personas con Diabetes, está integrada por cuatro conceptos. Las Conductas familiares y la Autogestión de las personas con diabetes tipo 2, son los principales, estos explican la interacción que se da entre ellas. El tercer concepto, Control glucémico, es la respuesta lograda entre la interación de los dos conceptos antes mencionados. Y por último, el cuarto concepto son los Agentes condicionantes individuales, mismo que se relaciona tanto con el concepto de las conductas, la persona con diabetes y el control glucémico.

\section{Conductas Familiares}

Las Conductas Familiares (CF) es el principal concepto de esta Teoría Específica (TE). Se describe como aquellos comportamientos realizados por parte de los miembros de la familia hacia la persona Diabetes tipo 2 (PDT2). Estas conductas pueden ser de dos tipos: efectivas e inefectivas.

En las CF efectivas, es donde los miembros de la familia proporcionan diferentes formas de apoyo (Informativo, emocional, instrumental y evaluativo), el cual contribuye a que la PDT2, lleve conductas favorables para controlar la enfermedad. Por otro lado, en las CF inefectivas, los miembros de la familia sabotean o socavan los esfuerzos de las personas con DT2, para realizar sus actividades; esto planificando y tentando a la PDT2 a consumir alimentos poco saludables, a no realizar actividad física, entre otras. La literatura ha reportado que el apoyo familiar influye de manera directa (positiva o negativamente) en las PDT2 para llevar a cabo el afrontamiento, la autogestión y la autoeficacia de la enfermedad y de manera indirectamente en el control metabólico de la enfermedad $(11,19,40)$.

\section{Autogestión de las personas con Diabetes tipo 2}

Es el segundo concepto principal de está TE. Se describe como aquella capacidad de la PDT2, para llevar acciones que le ayuden a manejar, controlar y resolver las situaciones propias de la enfermedad, de acuerdo con las recomendaciones de los profesionales de salud (Medico, Enfermera, Psicólogo, Nutriólogo, entre otros). Entre las acciones que debe de realizar la persona con DT2, se encuentra: a) el manejo de hipoglucemias e hiperglucemias, b) el cuidado de los pies, c) mejorar sus patrones de alimentación, d) práctica de actividad física, f) apego a medicación prescrita, g) abandono del consumo de alcohol y tabaco, h) manejo del estrés, y de complicaciones y sus efectos secundarios.

Se ha encontrado que existen dos elementos importantes por parte de la persona para lograr una Autogestión efectiva. El primero es la autoeficacia, la evidencia empírica reporta que es un elemento esencial e importante para que las PDT2 lleven a cabo el cambio de comportamientos de manera continua, de acuerdo a las demandas de la enfermedad y poder asegurar un control metabólico a corto y largo plazo(15,19,20). El segundo es el afrontamiento de la enfermedad es de suma importancia para llevar a cabo los cambios de conducta que favorecerán el control metabólico de la enfermedad ${ }^{(9,12)}$. Existe evidencia de que el afrontamiento emocional influye en el incremento o disminucion de las conductas de autogestión ${ }^{(9,11)}$. Por otra parte el afrontamiento centrado en el problema ha contribuido a mejorar las conductas de comportamiento para controlar la enfermedad(9).

\section{Control glucémico}

El control glucémico se describe como el resultado de las acciones llevadas a cabo por parte de la persona con DT2 y su familiar para controlar la enfermedad. Este se ve reflejado en los parámetros aceptables de Hemoglobina glucosilada ( $\mathrm{HbA} 1 \mathrm{C})$ y el control de la glucosa en sangre. Estudios reportan que las personas que llevan a cabo un óptimo Control glucémico, son aquellas que realizan acciones efectivas para modificar patrones de vida, las que cuentan con un apoyo familiar efectivo ${ }^{(10,41)}$.

\section{Agentes condicionantes individuales}

Los Agentes Condicionantes Individuales (ACl), se describe como aquellos agentes individuales que influyen directa o indirectamente para llevar a cabo las conductas de apoyo familiar, la autogestión y el control glucémico. Entre estos factores se encuentran el nivel educativo, el estatus socioeconómico, el conocimiento de 
la enfermedad, la calidad de vida, el grado de depresión y nivel de estrés, entre otros. Algunos $\mathrm{ACl}$ que se han logrado identificar por parte de los familiares, es el nivel de conocimiento y la habilidad del familiar para el manejo y control del padecimiento de su familiar; la persuación o presión ejercida por parte del familiar, el tener una relación de pareja y el genero ${ }^{(20,40)}$.

Los $\mathrm{ACl}$ que se han identificado en las PDT2 para llevar a cabo la autogestión, es el nivel educativo, el desconocimiento de la enfermedad, falta de apoyo familiar, la aceptación de la enfermedad, la calidad de vida, el nivel de estrés y depresión, la presencia de complicaciones, el tratamiento, el género, el contar con una pareja y el número de medicamentos ${ }^{(9,12,42,43)}$. Esta información se muestra en la Figura 2. Modelo de la Teoría Específica sobre las Conductas familiares para la Autogestión y Control glucémico de las personas con Diabetes tipo 2.

Figura 2. Modelo sobre la Teoría especifica sobre las Conductas familiares para la Autogestión y el control glucémico de las personas con Diabetes Tipo 2.

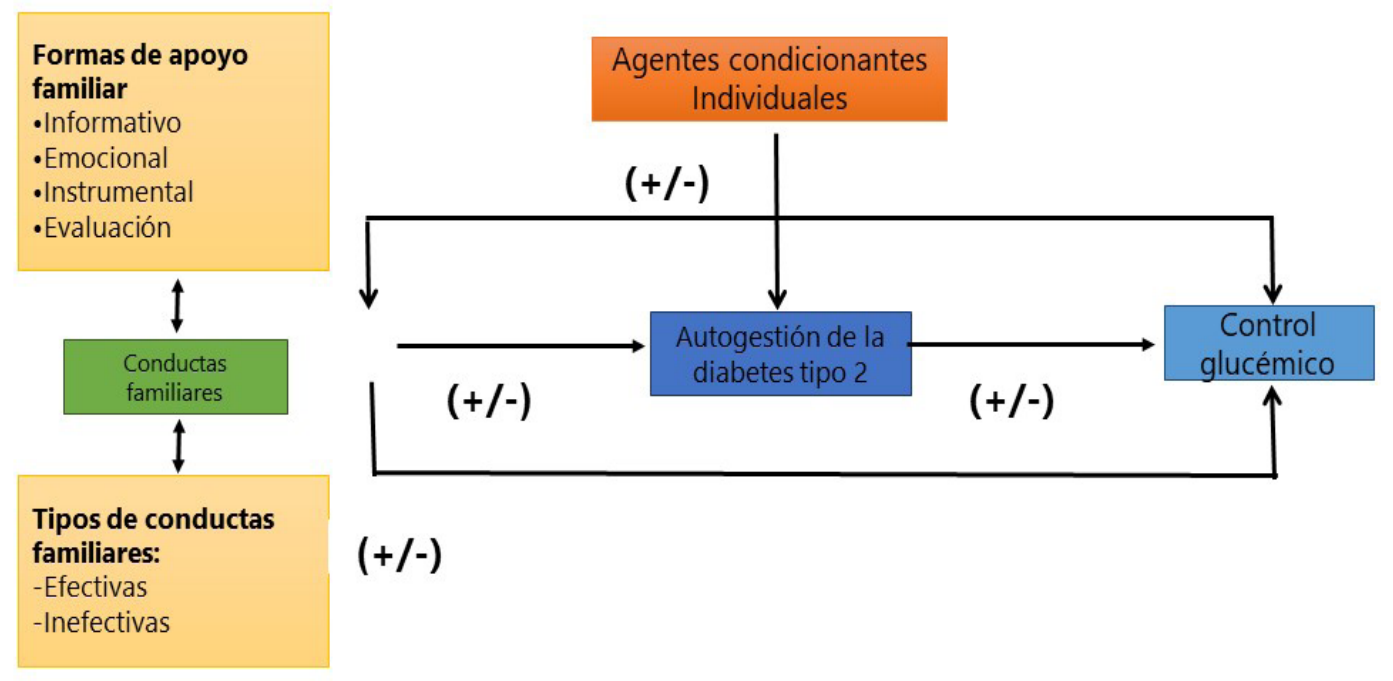

Fuente: Elaboracion propia

\section{Implicaciones en la practica de enfermeria}

Con base a lo analizado y descrito anteriormente respecto a la presente microteoría, se brindan algunas implicaciones para la práctica y la investigación. El profesional de enfermería y los diferentes proveedores de la salud, deben tomar en cuenta el contexto, la interacción y funcionamiento familiar de la persona que padece la Diabetes tipo 2.

Al mismo tiempo se debe valorar la capacidad para tomar decisiones y la autoconfianza de las personas con diabetes, ya que estos condicionan que la persona con lleve a cabo la autogestión de la enfermedad. En relación con investigación, se podrán incorporar los conceptos y su interrelación para proyectos de intervención donde se promueva la autogestión y el control glucémico, tomando en cuenta el contexto familiar.

Para un mayor desarrollo y adecuación funcional de la microteoría, se requiere probar y validar, a través de un estudio piloto o de intervención. Además se debe validar en otros grupos de edad y en diferentes entornos, ya que se diseñó para un grupo de edad específico y una estructura social. También se debe iniciar el desarrollo de indicadores empíricos específicos para cada concepto establecido.

\section{CONCLUSIONES}

La presente microteoría proporciona un marco teórico que explica las conductas de la familia en la Autogestión y Control glucémico de las personas con DT2, y cómo existen agentes que condicionan esta interacción. Ésta se puede aplicar en el entorno de la práctica clínica e investigación de enfermería y permite tener un marco de referencia para el desarrollo de intervenciones y programas de educación para lograr un control glucémico estricto en las personas con diabetes.

\section{REFERENCIAS BIBLIOGRÁFICAS}

1. American Diabetes Association. Standards of Medical Care in Diabetes-2018. Diabetes Care [Internet]. 
2018;41(Supplement 1):S1-156. Available from: http:// care.diabetesjournals.org/content/41/Supplement_1

2. Organización Mundial de la Salud. Informe Mundial sobre la diabetes: Resumen de orientación [Internet]. 2016. Available from: http://www.who.int/diabetes/ global-report/es/

3. International Diabetes Federation. Diabetes Atlas de la FID [Internet]. 8va ed. Diabetes Atlas IDF. Fderation International of Diabetes; 2015. 1-527 p. Available from: http://www.diabetesatlas.org/resources/2015-atlas. html\%5Cnhttps://www.idf.org/sites/default/files/EN_6E_ Atlas_Full_0.pdf\%5Cnwww.ecuadorencifras.gob.ec

4. Shamah-Levi T,Cuevas L, Dommarco J HM. Encuesta Nacional de Salud y Nutrición de Medio Camino 2016. (ENSANUT MC 2016). Inst Nac Salud Pública [Internet]. 2016; Available from: https://www.gob.mx/salud/ documentos/encuesta-nacional-de-salud-y-nutricionde-medio-camino-2016

5. Seuring $T$, Goryakin $Y$, Suhrcke $M$. The impact of diabetes on employment in Mexico. Econ Hum Biol [Internet]. 2015;18:85-100. Available from: https://www.sciencedirect.com/science/article/pii/ S1570677X15000349?via\%3Dihub

6. Sendhilkumar $M$, Tripathy JP, Harries AD, Dongre AR, Deepa $M$, Vidyulatha $A$, et al. Factors associated with high stress levels in adults with diabetes mellitus attending a tertiary diabetes care center, Chennai, Tamil Nadu, India. Indian J Endocrinol Metab [Internet]. 2017; 21(1):56-63. Available from: http://www.ijem.in/article.asp?issn $=2230$ 8210 ; year $=2017$; olume $=21$; issue $=1$; spage $=56$; epage $=6$ 3;aulast=Sendhilkumar

7. Xiang Y, Luo P, Cai X, Tang Y, Wu Z. Results of a pilot study of patient-to-patient education strategy on selfmanagement among glycemic uncontrolled patients with diabetes. Patient Prefer Adherence [Internet]. 2017; 11:787-793. Available from: https://www.dovepress. $\mathrm{com} /$ results-of-a-pilot-study-of-patient-to-patienteducation-strategy-on-s-peer-reviewed-article-PPA

8. Whitehead LC, Crowe MT, Carter JD, Maskill VR, Carlyle $D$, Bugge $C$, et al. A nurse-led interdisciplinary approach to promote self-management of type 2 diabetes: a process evaluation of post-intervention experiences. J Eval Clin Pract [Internet]. 2017; 23(2):264-71. Available from: https://onlinelibrary.wiley.com/doi/abs/10.1111/ jep.12594

9. Albai A, Sima A, Papava I, Roman D, Andor B, Gafencu M. Association between coping mechanisms and adherence to diabetes-related self-care activities: A cross-sectional study. Patient Prefer Adherence [Internet]. 2017; 11 Pages 1235-41. Available from: https://www.dovepress. com/association-between-coping-mechanisms-andadherence-to-diabetes-relate-peer-reviewed-article-PPA 10. Kir M, Ugurlu B, Unal N, Metin K, Yilmaz N, Kizilca O. Depression, coping strategies, glycemic control and patient compliance in Type 2 diabetic patients in an endocrine outpatient clinic. Pakistan J Med Sci [Internet].
2015;31(1):19-24. Available from: http://10.0.49.125/ pjms.311.6011\%5Cnhttp://ezproxy.spu.edu/ login?url=http://search.ebscohost.com/login.aspx?direct $=$ true $\&$ AuthType $=i p \& d b=a p h \& A N=108758962 \&$ site $=e$ host-live

11. Shayeghian $Z$, Aguilar-Vafaie $M E$, Besharat $M A$, Amiri $P$, Parvin $M$, Gillani $K R$, et al. Self-care activities and glycated haemoglobin in Iranian patients with type 2 diabetes: Can coping styles and social support have a buffering role? Psychol Heal. 2015; 2015;30(2):153-64.

12. Kokoszka A. Treatment adherence in patients with type 2 diabetes mellitus correlates with different coping styles, low perception of self-influence on disease, and depressive symptoms. Patient Prefer Adherence [Internet]. 2017;11:587-95. Available from: https://www. dovepress.com/treatment-adherence-in-patients-withtype-2-diabetes-mellitus-correlat-peer-reviewed-articlePPA

13. Vissenberg C, Nierkens V, Van Valkengoed I, Nijpels G, Uitewaal P, Middelkoop B, et al. The impact of a social network based intervention on self-management behaviours among patients with type 2 diabetes living in socioeconomically deprived neighbourhoods: A mixed methods approach. Scand J Public Health [Internet]. 2017; 45(6):569-583. Available from: http://journals. sagepub.com/doi/abs/10.1177/1403494817701565?url ver $=$ Z39.88-2003\&rfr_id $=$ ori:rid:crossref.org\&rfr_dat $=\mathrm{cr}_{\text {_ }}$ pub\%3Dpubmed

14. Tanash Ml, Fitzsimons D, Coates V, Deaton C. An evaluation of the effectiveness of self-management interventions for people with type 2 diabetes after an acute coronary syndrome: a systematic review. J Clin Nurs. 2017;26(11-12):1458-72.

15. Devchand R, Nicols C, Gallivan JM, Tiktin M, KrauseSteinrauf $H$, Larkin $M$, et al. Assessment of a National Diabetes Education Program diabetes management booklet: The GRADE experience. J Am Assoc Nurse Pract. 2017; 29(5):255-263.

16. Felton AM, LaSalle J, McGill M, Aschner P, Bailey C, Blonde $L$, et al. Treatment urgency: The importance of getting people with type 2 diabetes to target promptly. Diabetes Res Clin Pract. 2016; 117:100-3.

17. Smith-Miller CA, Berry DC, DeWalt D, Miller CT. Type 2 Diabetes Self-management Among Spanish-Speaking Hispanic Immigrants. J Immigr Minor Heal. 2016; 18(6):1392-1403.

18. Wichit N, Mnatzaganian G, Courtney M, Schulz P, Johnson M. Randomized controlled trial of a familyoriented self-management program to improve selfefficacy, glycemic control and quality of life among Thai individuals with Type 2 diabetes. Diabetes Res Clin Pract. 2017; 123:37-48.

19. Bhandari P, Kim M. Self-Care Behaviors of Nepalese Adults With Type 2 Diabetes: A Mixed Methods Analysis. Nurs Res. 2016; 65(3):202-14.

20. ALAboudi IS, Hassali MA, Shafie AA, Saleem F. Self- 
efficacy, self-care behaviours and glycaemic control in type 2 diabetic patients in Riyadh, Saudi Arabia. J Public Health (Bangkok). 2016; 24(1): 281-90.

21. Cohen S, McKay G. Social Support, Stress and the Buffering Hypothesis: A Theoretical Analysis. In: Handbook of Psychology and Health. 1984. p. 253-67.

22. Leahy-Warren P. Social Support Theory. In: Springer Publishing Company, editor. Practice Theories Guiding Nursing Research and Practice: Making Nursing Knowledge Development Explicit. 2014. p. 85-101.

23. Hinson Langford CP, Bowsher J, Maloney JP, Lilis PP. Social support: a conceptual analysis. J Adv Nurs [Internet]. 1997;25:95-100. Available from: https:// onlinelibrary.wiley.com/doi/abs/10.1046/j.13652648.1997.1997025095.x

24. Schaffer MA. Social Support. In: Peterson SJ, Bredow TS, editors. Middle Range Theories Aplication to Nursing Research. 3ra ed. Wolters Kluwer Lippincott Williams \& Wilkins; 2014. p. 108-27.

25. Mayberry LS, Harper KJ, Osborn CY. Family behaviors and type 2 diabetes: What to target and how to address in interventions for adults with low socioeconomic status. Chronic Illn. 2016; 12(3):199-215.

26. Bahremand $M$, Rai $A$, Alikhani $M$, Mohammadi $S$, Shahebrahimi K, Janjani P. Relationship Between Family Functioning and Mental Health Considering the Mediating Role of Resiliency in Type 2 Diabetes Mellitus Patients. Glob J Health Sci. 2014; 7(3):254-9.

27. Thojampa S, Mawn B. The moderating effect of social cognitive factors on self-management activities and $\mathrm{HbA1c}$ in Thai adults with type-2 diabetes. Int J Nurs Sci. 2017; 4(1):34-7.

28. Meleis Al. Theoretical Nursing Development \& Progress. 5th ed. Philadelphia: Wolters Kluwer Lippincott Williams \& Wilkins; 2012. 672 p.

29. Grove SK, Burns N, Gray JR. The Practice of Nursing Research appraisal, Synthesis, and Generation of Evidence. 7th ed. St. Louis, Missouri: Elsevier; 2013. 732 p.

30. Walker LO, Avant KC. Strategies for Theory Construction in Nursing. Boston: Prentice Hall. 2011.

31. Im E-O, Meleis Al. Situation- specific Theories: Philosophical Roots, Properties, and Approach. Adv Nurs Sci [Internet]. 1999;22(2):11-24. Available from: https://journals.Iww.com/advancesinnursingscience/ Abstract/1999/12000/Situation_Specific_Theories_Philosophical_Roots,.3.aspx

32. Im E-O. Development of Situation-specific Theories An Integrative Approach. Adv Nurs Sci [Internet]. 2005;28(2):287-8. Available from: https://journals.lww. com/advancesinnursingscience/Abstract/2005/04000/ Development_of_Situation_specific_Theories_An.6.aspx 33. Finfgeld-connett D. Clarification of Social Support. J Nurs Scholarsh [Internet]. 2005;37(1):4-9. Available from: https://sigmapubs.onlinelibrary.wiley.com/doi/ abs/10.1111/j.1547-5069.2005.00004.x

34. Cobb S. Social support as a moderator of life stress.
Psychosom Med [Internet]. 1976;38(5):300-14. Available from: http://www.ncbi.nlm.nih.gov/pubmed/981490

35. Heaney CA, Barbara A I. Social networks and social support. In: Glanz K, Rimer BK, Viswanath K, editors. Health Behavior and Health Education. 5ta ed. JOSSEYBASS A Wiley Brand; 2008. p. 189-200.

36. Schafer LC, McCaul KD, Glasgow RE. Supportive and nonsupportive family behaviors: Relationships to adherence and metabolic control in persons with type I diabetes. Diabetes Care. 1986; 9(2): 179-85.

37. Smith MJ, Liehr PR. Middle Range Theory for Nursing. 3rd ed. New York: Springer Publishing company; 2014. $443 \mathrm{p}$.

38. Bartholomew Eldrigde, L. K., Markham, C. M., Ruiter, R. A. C., Fernàndez, M. E., Kok, G., \& Parcel GS. Planning health promotion programs: An Intervention Mapping approach. 4th ed. Planning health promotion programs An intervention mapping approach. JOSSEY- BASS A Wiley Brand; 2016.

39. Ramkisson S, Pillay BJ, Sibanda W. Social support and coping in adults with type 2 diabetes. African J Prim Heal Care Fam Med [Internet]. 2017;9(1):1-8. Available from: http://www.phcfm.org/index.php/phcfm/article/ view/1405

40. Shi M, Xu M-Y, Liu Z-L, Duan X-Y, Zhu Y-B, Shi H-M, et al. Effectiveness of family involvement in newly diagnosed type 2 diabetes patients: a follow-up study. Patient Educ Couns. 2016; 99(5):776-82.

41. Huang M, Zhao R, Li S, Jiang X. Self-management behavior in patients with type 2 diabetes: A cross-sectional survey in western urban China. PLoS One. 2014;9(4).

42. Kim C-J, Schlenk EA, Kim DJ, Kim M, Erlen JA, Kim $\mathrm{S}-\mathrm{E}$. The role of social support on the relationship of depressive symptoms to medication adherence and selfcare activities in adults with type 2 diabetes. J Adv Nurs [Internet]. 2015; 71(9): 2164-75 Available from: https:// onlinelibrary.wiley.com/doi/10.1111/jan.12682

43. Ashur ST, Shah SA, Bosseri S, Fah TS, Shamsuddin K. Glycaemic control status among type 2 diabetic patients and the role of their diabetes coping behaviours: A clinic-based study in Tripoli, Libya. Libyan J Med. 2016; 11:31086. 